\title{
Epidural blood patch under fluoroscopic control: non-surgical treatment of lumbar cerebrospinal fluid fistula following implantation of an intrathecal pump system
}

\author{
$\mathrm{K} \mathrm{Huch}^{*}{ }^{1}$, U Kunz ${ }^{2}$, P Kluger ${ }^{1}$ and W Puhl ${ }^{1}$ \\ ${ }^{1}$ Department of Orthopedic Surgery with Spinal Cord Injury Unit, University of Ulm, Oberer Eselsberg 45, Germany; \\ ${ }^{2}$ Department of Neurosurgery, Military Hospital Ulm, Oberer Eselsberg 40, Germany
}

\begin{abstract}
The treatment of lumbar cerebrospinal fluid fistula in the presence of an intrathecal catheter is known to be difficult. Open revision surgery is recommended in the literature, although the rate of recurrence is high. The epidural blood patch technique is well established as a successful treatment for post-dural-puncture headaches. Recent work about the distribution of the injected blood and theoretical considerations about the mechanism of action make this method suitable for the occlusion of spinal leakage even in the presence of an intrathecal catheter. In this note technical details are given for a successful therapy of lumbar cerebrospinal fluid fistula including the right positioning of the opening of the needle (cerebrospinal fluid can be expected intrathecally and epidurally) by injection of contrast medium first for myelography then for epidurography. In this procedure the (epidural) distribution of autologous blood can be indirectly controlled by compression of the dural sac. The method is easy to perform, and the possible risks are small.
\end{abstract}

Keywords: epidural blood patch; fluoroscopy; cerebrospinal fluid fistula; intrathecal pump; non-surgical treatment

\section{Introduction}

Since 1984 when Penn and Kroin ${ }^{1}$ first described the therapy of severe spasticity with continuous intrathecal baclofen this procedure became popular because of its benefits and became a less invasive alternative to destructive neurosurgical procedures such as myelotomies and anterior rhizotomies. ${ }^{2}$ Advantages include the possibility of long-term treatment, ${ }^{2}$ selective delivery of baclofen with less systemic side effects, and constant drug levels with continuous reduction of spasticity. However, this method is not free of drawbacks. The delivery pumps are expensive, infections of the pump pocket or meningitis may occur, and technical problems (kinking or disconnection of a tube, and malfunction of programmable pumps) are possible. Another possible complication is the development of spinal headaches. Recently, Rauck et $a l^{3}$ reported a case of symptomatic paraspinal fluid extravasation diagnosed 2 years after implantation of an intrathecal catheter system.

Over the last decades anesthesiologists have used the epidural blood patch technique as a routine to relieve post dural puncture headache, ${ }^{4-7}$ Nitescu et $a l^{8}$ performed the epidural blood patch as a successful treatment of postdural puncture headache occurring after implantation of an intrathecal catheter.

*Correspondence: K Huch, MD, Department of Orthopedic Surgery, RKU/University of Ulm, Oberer Eselsberg 45, 89081 Ulm, Germany
As another complication of intrathecal catheter implantation internal leakage of cerebrospinal fluid can cause the formation of a subcutaneous hygroma ('pseudomeningocele'). ${ }^{18}$ This was observed by Nitescu et $a l^{8}$ in $1.5 \%$ of the patients 8,27 and 53 days after insertion of the intrathecal catheter, whereas Strong 9 reported a rate of $4 \%$ and Krames et $a l^{10}$ a rate of $6 \%$. Consequences include complicated refilling of the pump, drug dilution and possible wound breakdown leading to a cutaneous fistula of cerebrospinal fluid. ${ }^{11}$ With open leakage of cerebrospinal fluid, complete wound debridement and surgical repair is recommended by Eismont et al ${ }^{12}$ and Nitescu et al. ${ }^{8}$ Small hygromas can be treated by a simple compressive dressing at the insertion site. ${ }^{8}$ Aspiration drainage of large hygromas is recommended. ${ }^{8}$ However, Spahn and Müller ${ }^{13}$ described two cases in which a single aspiration of the cerebrospinal fluid led to a spontaneous closure of the dural leakage with the intrathecal catheter in situ.

As an alternative to aspiration or surgical intervention an epidural blood patch was used in some cases to stop cerebrospinal fluid leakage. ${ }^{14,15}$ Only one case report describes the successful treatment of a cerebrospinal fluid cutaneous fistula by an epidural blood patch in the presence of an intrathecal drug delivery system. ${ }^{16}$ However, the procedure was performed without visualization by fluorography or contrast. In a recent technical case report Chauhan et 
$a l^{17}$ described the successful application of an epidural blood patch after removal of an intrathecal pump system or a lumbar peritoneal shunt.

The technique of the non-surgical treatment of subcutaneous spinal fluid hygroma occurring after implantation of an intrathecal catheter-pump-system using an epidural blood patch is described. An easy method for the exact localization of the epidural space with the needle and for visualization of the blood patch size is presented together with technical details which appear important for the success of the procedure.

\section{Methods}

In 1983 the thoracolumbal scoliosis of a now 32-year old patient with $\mathrm{M}$ Recklinghausen was corrected according to Harrington's procedure. In the same year a large neurofibroma next to the spine was removed in another surgical procedure and a ventral spondylodesis with a fibular bone graft was achieved. Postoperatively the patient suffered from a paraplegia below Th6 and subsequently developed severe spasticity. Oral application of baclofen caused various side effects. Therefore, intrathecal injections were tried. Since injection of $50 \mu \mathrm{g}$ baclofen twice a day effectively reduced his spasticity without side effects, the implantation of an intrathecal pump system (Infusaid ${ }^{\circledR}$ ) was offered to the patient and he was informed in detail about possible risks and complications. ${ }^{18}$

A 14-gauge Tuohy needle was inserted into the subarachnoid space at L4-L5 and a $1.6 \mathrm{~mm}$-catheter was inserted through this needle. After removal of the needle the catheter was tunneled subcutaneously around the waist and a subcutaneous pocket for the pump was prepared in the right lower abdominal wall. Pump and catheter were connected and fixed by sutures. On day 6 after the implantation a large subcutaneous hygroma of cerebrospinal fluid developed around the catheter and the pump. To confirm the diagnosis contrast medium was injected via the side-port of the Infusaid $^{\circledR}$ pump performing a myelogram and demonstrating the leakage along the implanted catheter (Figure 1). Three times between 300 and $400 \mathrm{ml}$ of a clear yellowish fluid without any sign of infection could be aspirated percutaneously around the pump. After each drainage a compressive dressing was put around the waist. Because of the risk of infection and meningitis the aspiration manoevres were stopped. The effect of baclofen disappeared, most likely due to dilution by a volume, which was approximately three times higher than the normal volume $(120-200 \mathrm{ml}$ cerebrospinal fluid).

Since surgical revision of cerebrospinal fluid fistulae is accompanied by a high rate of recurrence the epidural blood patch technique was favored as a less invasive method. First, the cerebrospinal fluid in the subcutaneous pouch was drawn via a 14-gauge needle under aseptic conditions. Then a spinal needle (end- hole) was inserted via the interspinous space below the catheter entrance (L5-S1) to avoid laceration of the intrathecal catheter (Figure 1). CSF was aspirated and $5 \mathrm{ml}$ contrast medium were injected to localize the tip of the needle (Figure 2), because CSF was expected not only subarachnoidally but also epidurally after catheter implantation and after various other spinal procedures in the past. Following this myelogram (Figure 2) the needle was retracted under aspiration

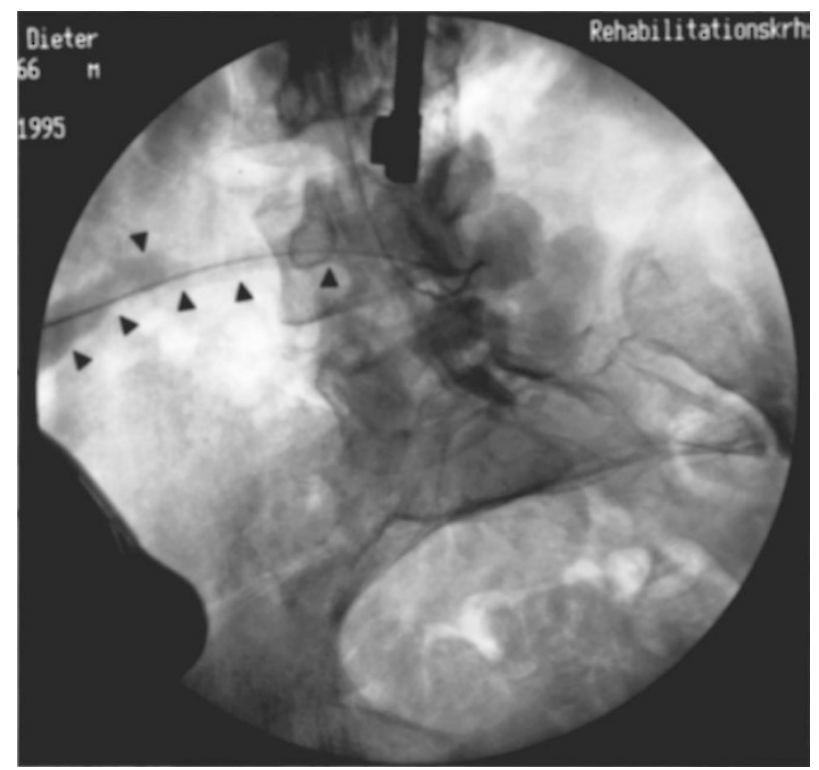

Figure 1 Myelography (via side port of the intrathecal pump) showing the CSF-fistula next to the intrathecal catheter

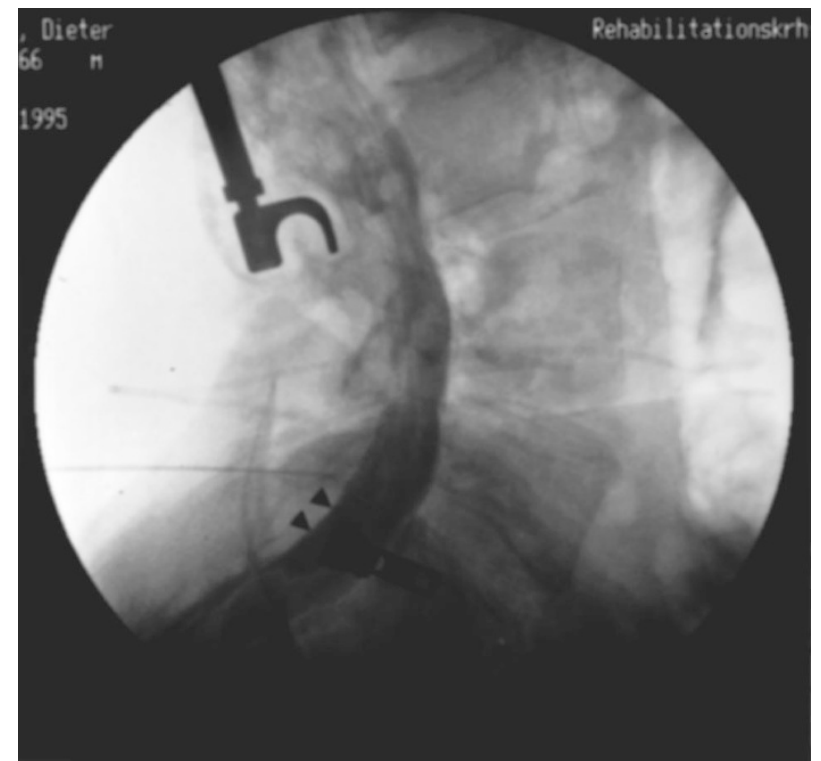

Figure 2 Positioning of the needle directly after the myelography (see Figure 1) 
then another $1 \mathrm{ml}$ of contrast medium was injected performing an epidurogram. ${ }^{19}$ Keeping the needle in a stable position, autologous blood $(15 \mathrm{ml}$ taken under aseptic conditions) was injected via a three way adapter into the epidural space. The expansion of the blood patch was visualized by the next radiograph (Figure 3). There were no symptoms during the injection. Six weeks after the procedure the patient developed a small recurrence of his subcutaneous CSF cyst which has shown a steady state over 3 years, so that the spasticity could effectively be reduced by intrathecal baclofen administration via the pump.

\section{Results and discussion}

The main indication for percutaneous injection of an epidural blood patch is the post lumbar puncture headache. This headache is thought to be caused by a leakage of cerebrospinal fluid after puncture of the dura eg for spinal anesthesia. However, some authors used this technique to seal cerebrospinal fluid fistulae following laminectomy ${ }^{20}$ or other surgical procedures, ${ }^{14,15,21}$ avoiding surgical revision which has been the traditional way of treatment. ${ }^{12}$ Only one report described the successful treatment of an open (cutaneous) cerebrospinal fluid fistula by an epidural blood patch in the presence of an intrathecal catheter. $^{16}$ No details about the right positioning of the needle in the epidural space are given in this report.

Epidural blood placement appears to be very effective in the treatment of post-lumbar-puncture headache. $^{22}$ The injection of blood into the epidural space starts an interaction between blood and cerebrospinal fluid leading to a clot reaction which is four times faster than an activated clotting time. ${ }^{23}$ An

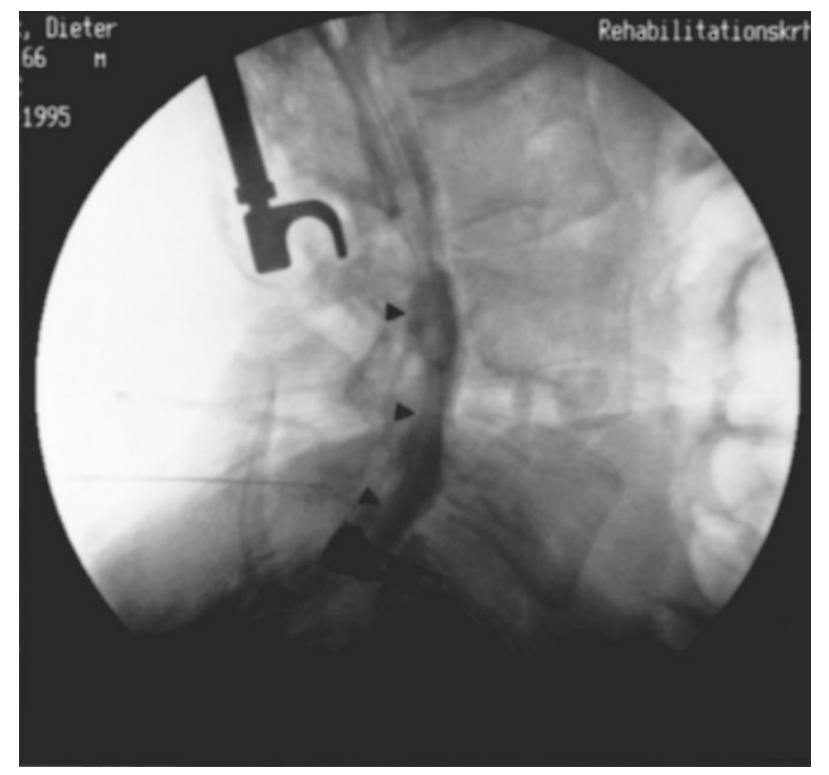

Figure 3 Extension of the blood patch visualized by impression of the dural sac in vitro study showed that this plug can withstand normal cerebrospinal fluid pressures in patients in the sitting position. The same authors speculated that injury of the endothelial like dura promotes coagulation by mechanisms similar to those associated with vascular damage. ${ }^{24}$ An autopsy of an obstetric patient demonstrated that a 3-day old epidural blood patch consisted of clots adherent to the lumbar and sacral dura. $^{25}$ Histologically this blood clot formation is followed by a fibrous reaction which reaches its maximum during the first 3 weeks. ${ }^{26}$ The blood is widely spread within the epidural space. Vakharia et $a l^{27}$ performed a blood patch with $20 \mathrm{ml}$ in five patients and using magnetic resonance imaging they demonstrated a mean spread over 4.6 intervertebral spaces. This spread is principally more upwards than downwards from the injection site. ${ }^{19,28,29}$ Therefore, the blood should be injected into the epidural space below the original level of the spinal puncture. ${ }^{29}$

Recommendations for the amount of autologous blood needed to perform an epidural patch vary between 2 and as much as $30 \mathrm{ml}$ (Table 1). The injection should be slow and stopped if the patient complains about discomfort. ${ }^{29}$ Contrary to recent studies we performed our extradural puncture after localizing the extradural space by withdrawing the needle after initial subarachnoid spinal puncture according to Gormley's original publication in $1960{ }^{4}$ In addition we performed a myelogram and an epidurogram to confirm the position of the needle tip. We injected a volume of about $15 \mathrm{ml}$, representing the median volume of the papers reported in Table 1, without clinical symptoms; we demonstrated a more cranial distribution of the injected blood (Figure 3) as observed by others ${ }^{28,29}$ and an effective thecal compression in the entrance region of the intrathecal catheter as previously described by Carrie. ${ }^{30}$ The use of radiographic imaging was especially chosen because our patient had cerebrospinal fluid also in the epidural

Table 1 Amounts of blood recommended or used by different authors for epidural blood patching (only names of first authors mentioned)

\begin{tabular}{|c|c|}
\hline Abouleish $\mathrm{E}^{33}$ & $7-10 \mathrm{ml}$ \\
\hline Andrews PJD $^{39}$ & $15 \mathrm{ml}$ \\
\hline Barbera-Alacreu $\mathrm{M}^{15}$, Kumar $\mathrm{V}^{40}$ & $17-22 \mathrm{ml}$ \\
\hline Beards SC $^{29}$ & $18-20 \mathrm{ml}$ \\
\hline Chauhan $\mathrm{C}^{17}$ & $20-30 \mathrm{ml}$ \\
\hline Crawford $\mathrm{JS}^{41}$ & $20 \mathrm{ml}$ \\
\hline DiGiovanni $\mathrm{AJ}^{42}$ & $5-10 \mathrm{ml}$ \\
\hline Gormley $\mathrm{JB}^{4}$ & $2-3 \mathrm{ml}$ \\
\hline Hardy PAJ $^{16}$ & $20 \mathrm{ml}$ \\
\hline Howes $\mathrm{J}^{43}$ & $10-20 \mathrm{ml}$ \\
\hline Maycock $\mathrm{NF}^{20}$ & $\begin{array}{c}25 \mathrm{ml} \\
\text { (used after laminectomy) }\end{array}$ \\
\hline Nitescu $\mathrm{P}^{8}$ & $15-20 \mathrm{ml}$ \\
\hline Odzil $\mathrm{T}^{44}$ & $2.5 \mathrm{ml}$ \\
\hline Seebacher ${ }^{45}$ & $10-20 \mathrm{ml}$ \\
\hline Szeinfeld $\mathbf{M}^{28}$ & $12-18 \mathrm{ml}$ \\
\hline
\end{tabular}


space, because the anatomy was changed after different surgical interventions in the lumbar spine, and because an injection at the wrong level is believed to be a major reason for the failure of blood patching. ${ }^{29}$ Instantaneous compression of the thecal sac as demonstrated in Beards' et $a l^{29}$ and in our study together with a rapid increase in cerebrospinal fluid pressure could explain the rapid response of post lumbar puncture headache to epidural blood patching. As mentioned above we used a spinal end-hole needle because Beards et $a l^{29}$ observed in their magnetic resonance imaging study of extradural blood patches that a considerable volume of blood extended from the site of insertion of the Tuohy needle into fascial planes between the subcutaneous fat compartments. These authors speculated that using the loss of resistance technique for the localization of the epidural space could lead to a position of the needle opening only partly in this space when the blood is injected. ${ }^{29}$ This could explain at the same time that smaller volumes $(6-10 \mathrm{ml})$ led to a failure in as many as $25 \%$ of the patients if a Tuohy needle was used. $5,22,31,32$

In general, complications are rarely seen in blood patching and are usually self-limiting. Abouleish et $a l^{33}$ described the following: during the procedure $2 \%$ of the patients developed backache, $1 \%$ each paraesthesia, neckache or dural puncture. Within $48 \mathrm{~h}$ after an epidural blood patch, 35\% complain about backache, 5\% about transient (lasting $12-24 \mathrm{~h}$ ) temperature elevation of $1{ }^{\circ} \mathrm{C}$ and $1 \%$ about neckache. Radicular pain is a rarely reported complication of short duration ${ }^{31,34,35}$ possibly caused by nerve root compression through the extradural clot. ${ }^{29}$ One case report described an acute deterioration of mental status after epidural blood patching in a patient suffering from benign meningioma. After external ventriculostomy for obstructive hydrocephalus and total resection of the tumor the patient returned to his normal mental status. ${ }^{36}$ Fever, leucocytosis, back pain or neurological changes may be a sign of the development of an epidural abscess and should be followed up. ${ }^{9,37}$ In one case of six blood patches performed in a single patient for post-lumbar-puncture headache a spinal subdural epiarachnoid hematoma developed. ${ }^{38}$

$\mathrm{n}$ conclusion, the epidural blood patch technique can be used not only for the treatment of post lumbar puncture headache but can also be applied in a modified manner for the blockage of cerebrospinal fluid fistulae in the absence and even in the presence of an intrathecal catheter. The position of the needle for the autologous blood patch can be controlled by injection of contrast medium. Experience showed that the blood will more likely spread upwards so that the risk of intrathecal catheter damage can be minimized by an approach one segment below the entrance of the catheter. In a CSF-hygroma the epidural blood patch should be performed in time because a spontaneous skin breakdown would increase the risk of infection. This risk is especially high in the presence of silicone.
Additional uses of this technique may include a CSF-fistula beside a shunt or a cranioplasty with subcutaneous CSF-leakage.

\section{References}

1 Penn RD, Kroin JS. Continuous intrathecal baclofen alleviates spinal cord spasticity. Lancet 1984; 10: 1078.

2 Penn RD, Kroin JS. Long-term intrathecal baclofen infusion for treatment of spasticity. J Neurosurg 1987; 66: 181-185.

3 Rauck RL et al. Paraspinal fluid extravasation from long-term epidural catheter delivery system. Anesthesiology 1998; 88: $1672-1675$.

4 Gormley JB. Treatment of post-spinal headache. Anesthesiology 1960; 21: $565-566$.

5 Ostheimer GW, Palahniuk RJ, Shnider SM. Epidural blood patch for post-lumbar-puncture headache. Anesthesiology 1974; 41: $307-308$.

6 Dudziak R. Lehrbuch der Anästhesiologie. FK Schattauer: Stuttgart 1982, p 447.

7 Orkin FK, Cooperman LH. Complications in anaesthesiology. JB Lippincott: Philadelphia 1983, p 90.

8 Nitescu P, Sjöberg M, Appelgren L, Curelaru I.Complications of intrathecal opiods and bupivacaine in the treatment of 'refractory' cancer pain. Clin J Pain 1995; 11: 45-62.

9 Strong WE. Epidural abscess associated with epidural catheterization: a rare event? Report of two cases with marked delayed presentation. Anesthesiology 1991; 74: 943 -946.

10 Krames ES, Lanning RM. Intrathecal infusional analgesia for nonmalignant pain: analgesic efficacy of intrathecal opiod with or without bupivacaine. J Pain Symptom Manage 1993; 8: 539- 558

11 Waldman SD, Coombs DW. Selection of implantable delivery systems. Anesth Analg 1989; 68: $377-384$.

12 Eismont FJ, Wiesel SW, Rothman RH. Treatment of dural tears associated with spinal surgery. J Bone Joint Surg 1981; 63A: $1132-1136$.

13 Spahn B, Müller H. Behandlung der spinalen Spastik durch intrathekale Baclofen-Gabe. In: Grüninger W. (ed). Spinale Spastik. Ueberreutger, Wien 1989, pp 59-63.

$14 \mathrm{Katz}$ J. Treatment of a subarachnoid-cutaneous fistula with an epidural blood patch. Anesthesiology 1984; 60: 603-604.

15 Barbera-Alacreu M, Pallares VS. Tratamiento mediante parche hematico epidural de las fistulas de liquido cefalorraquideo. Rev Exp Anestesiol Reanim 1988; 35: 161 - 164.

16 Hardy PA. Extradural blood patch of a cerebrospinal fluid cutaneous fistula in the presence of an intrathecal drug delivery system. Anesthesiology 1994; 81: $1299-1300$.

17 Chauhan C, Francis GA, Kemeny AA. The avoidance of surgery in the treatment of subarachnoid cutaneous fistula by the use of an epidural blood patch: technical case report. Neurosurgery 1995; 36: $612-614$.

18 Coombs DW, Saunders RL, Gaylor M, Pageau MG. Epidural narcotic infusion reservoir: implantation technique and efficacy. Anethesiology 1982; 56: 469-473.

19 Burn JM, Guyer PB, Langdon L. The spread of solutions injected into the epidural space. A study using epidurograms in patients with the lumbosciatic syndrome. Br J Anaesth 1973; 45: 338 - 345.

20 Maycock NF, van-Essen J, Pfitzner J. Post-laminectomy cerebrospinal fluid fistula treated with epidural blood patch. Spine 1994; 19: $2223-2225$.

21 Lauer KK, Haddox JD. Epidural blood patch as treatment for a surgical durocutaneous fistula. J Clin Anesth 1992; 4: 45-47.

22 Bart AJ, Wheeler SA. Comparison of epidural saline placement and epidural blood placement in the treatment of post lumbar puncture headache. Anesthesiology 1978; 48: $221-223$.

23 Cook MA, Watkins-Pitchford JM. Epidural blood patch. A rapid coagulation response. Anesth Analg 1990; 70: 567-568.

24 Rosenberg PH, Heavner JE. In vitro study of the effect of epidural blood patch on leakage through a dural puncture. Anesth Analg 1985; 64: $501-504$. 
25 Edelman JD, Wingard DW. Subdural haematomas after lumbar dural puncture. Anesthesiology 1980; 52: 166-167.

26 DiGiovanni AJ, Galbert MW, Wahle WM. Epidural injection of autologous blood for postlumbar-puncture headache. Anesth Analg 1972; 51: 226-232.

27 Vakharia SB et al. Magnetic resonance imaging of cerebrospinal fluid leak and tamponade effect of blood patch in postdural puncture headache. Anesth Analg 1997; 84: 585-590.

28 Szeinfeld $\mathrm{M}$ et al. Epidural blood patch. Evaluation of the volume and spread of blood injected into the epidural space. Anesthesiology 1986; 64: 820-822.

29 Beards SC, Jackson A, Griffiths AG, Horsman EL. Magnetic resonance imaging of extradural blood patches: appearances from 30 min to 18 h. Br J Anaesth 1993; 71: 182-188.

30 Carrie LES. Epidural blood patch - why the rapid response? Anesth Analg 1991; 72: $129-130$.

31 Brownridge P. The management of headache after accidental dural puncture in obstetric patients. Anaesth Intens Care 1983; 11: $4-15$.

32 Loeser EA, Hill GE, Bennet GM, Sederberg JH. Time vs success rate for epidural blood patch. Anesthesiology 1978; 49: 147-148.

33 Abouleish E, De la Vega S, Blendinger I, Tio TO. Longterm follow-up of epidural blood patch. Anesth Analg 1975; 54: 459463.

34 Cornwall RD, Dolan WM. Radicular back pain following lumbar epidural blood patch. Anesthesiololgy 1975; 43: 692-693.

35 Shantha TR, McWhirter WR, Dunbar RW. Complications after epidural 'blood patch' for post lumbar-puncture headache. Anesth Analg 1973; 52: 67-72.
36 Beers RA, Cambreri JJ, Rodziewicz GS. Acute deterioration of mental status following epidural blood patch. Anesth Analg 1993; 76: $1147-1149$.

37 Priestley GS, Brighouse D. Cerebrospinal cutaneous fistula [letter]. Anaesthesia 1994; 49: 744.

38 Reynolds AF, Hameroff SR, Blitt CD, Roberts WL. Spinal subdural epiarachnoid hematoma: A complication of a novel epidural blood patch technique. Anesth Analg 1980; 59: 702 - 703 .

39 Andrews PJD, Ackerman WE, Juneja M, Cases-Cristobal V, Rigor BM. Transient bradycardia associated with extradural blood patch after inadvertent dural puncture in parturients. $\mathrm{Br} J$ Anaesth 1992; 69: $401-403$.

40 Kumar V, Maves T, Barcellos W. Epidural blood patch for treatment of subarachnoid fistula in children. Anaesthesia 1991; 46: $117-118$

41 Crawford JS. Experiences with epidural blood patch. Anaesthesia 1980; 35: $513-515$.

42 DiGiovanni AJ, Dunbar BS. Epidural injections of autologous blood for postlumbar-puncture headache. Anesth Analg 1970; 49: $268-271$

43 Howes J, Lenz R. Cerebrospinal fluid cutaneous fistula An unusual complication of epidural anaesthesia. Anaesthesia 1994; 49: $221-222$

44 Ozdil T, Powell WF. Post lumbar puncture headache: an effective method of prevention. Anesth Analg 1965; 44: $542-545$.

45 Seebacher $\mathbf{J}$ et al. Epidural blood patch in the treatment of post dural puncture headache: A double blind study. Headache 1989; 29: $630-632$. 